\title{
Applications of TEM and 3DAP to Measurement of Phase Diagrams
}

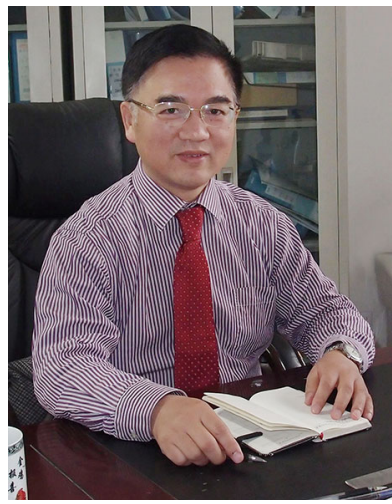

More than 40 years have passed since the birth of CALculation of PHAse Diagrams (CALPHAD) method when Larry Kaufman introduced the very important concept of "lattice stability" in his famous book entitled "Computer Calculation of Phase Diagrams" (Kaufman and Bernstein, Computer Calculation of Phase Diagrams, Academic Press, New York, 1970). Since then CALPHAD method has been widely used and further developed, and is now considered an important part of Integrated Computational Materials Engineering (ICME) (Integrated Computational Materials Engineering, National Academies Press, Washington DC, 2008) and an essential component of materials genomics (Kaufman and Ågren, Scripta Mater. 70, 2014, p 3).

The dominant thermodynamic quantity on which the CALPHAD method depends is the Gibbs energy. Using standard procedures, a large number of quantities, such as phase equilibria, enthalpy and entropy, can be calculated from Gibbs energy. The optimization of the Gibbs energy functions in a system relies on the experimental phase diagram and thermodynamic properties. When experimental data are limited or missing, atomistic calculations, such as first-principles calculations and molecular dynamics, could be employed to provide these data. With rapidly increasing computational power and development of sophisticated computational algorithms in the past decades, theoretical calculations are playing an increasingly important role in CALPHAD assessments, reducing the amount of the experiment work needed. However, no one doubts that experimental phase diagram and thermodynamic data are still needed to validate the calculated results. Accurate phase diagram and thermodynamic data are prerequisite for reliable CALPHAD modeling.

Nowadays, the experimental methods generally used to determine phase diagrams remain nearly the same as those utilized decades ago. For example, a general procedure to measure one isothermal section of a ternary system at one specified temperature is as follows. First, alloys are prepared via arc melting or induction melting or other sample preparation methods. Subsequently, the alloys are annealed at that temperature for extended periods of time to reach equilibrium state, followed by quenching. Finally, the alloys are analyzed by means of x-ray diffraction (XRD), electron probe microanalysis (EPMA), scanning electron microscopy (SEM) etc. to provide the phase equilibrium information for constructing the isothermal section. Other methods like diffusion couples along with SEM or EPMA have been developed to construct the isothermal sections using a smaller number of samples. However, classical experimental techniques, such as XRD, EPMA, and SEM, usually have limits measuring volume fraction or composition of phases. These techniques cannot provide reliable phase equilibrium information for very small particles. For example, it is problematic for XRD to detect the presence of a trace amount of a phase. SEM can hardly resolve particles of tens of nanometers in size, and EPMA cannot provide reliable composition information for phases with a size below about $1 \mu \mathrm{m}$. In order to remedy this situation, more advanced experimental techniques with higher resolution and sensitivity are needed. Transmission electron microscopy (TEM) and three-dimensional atom probe (3DAP) are two promising and powerful techniques meeting these demands. Here, I would like to present three case studies in which TEM or 3DAP in combination with traditional methods were successfully used to establish reliable phase diagrams of ternary or quaternary systems containing very small particles.

Case study one: Phase equilibria in the Al-rich corner of the quaternary Al-Fe-Ni-Si system (Hao et al., J. Mater. Sci. 49, 2014, p 1157)

The Al-Fe-Ni-Si quaternary system is of critical importance to aluminum industry since many commercial Al alloys contain $\mathrm{Fe}, \mathrm{Ni}$, and $\mathrm{Si}$ as impurities or alloying elements. However, information on phase equilibria and solidification behavior in the Al-rich corner of this quaternary system is rather limited in the literature. Using a hybrid approach of experiment (XRD, SEM, EPMA, DTA, TEM) and thermodynamic modeling, a self-consistent thermodynamic parameters for the Al-rich corner of the quaternary system were obtained. This permitted construction of a full map of phase equilibria and thermodynamic properties of the quaternary system over the targeted composition and temperature ranges It was found that $\mathrm{Al}_{8} \mathrm{Fe}_{2} \mathrm{Si}$ particles embedded in the (Al) matrix during solidification are of nano-scale. In order to capture the nano-size particles in as-cast samples, TEM was employed, which provides high resolution electron microscopy images of the nanosized particles at high magnification. Fast fourier transformation (FFT) patterns of the nanoparticles were derived and indexed to determine their crystal structure. Results from Gulliver-Scheil simulations were also compared with experimental microstructures of as-cast alloys. The 
simulations agree well with the experimental observations based on XRD, SEM and TEM. This would not have been possible if only results from XRD and SEM were used.

Case study two: Phase equilibria of Ag-Sb-Te thermoelectric materials (Wu and Chen, Acta Mater. 59, 2011, p 6463).

As indicated in the first case study, nanosized particles can be observed in the as-cast state. Nanosized precipitates can also be identified in annealed states, as was shown in determination of isothermal sections at 250 and $400{ }^{\circ} \mathrm{C}$ of the $\mathrm{Ag}$-Sb-Te system by means of XRD, EPMA and TEM. The phase relations that were obtained are of fundamental importance for investigating the effect of nanostructure and nanosized precipitates on thermoelectric properties. The innovative point in Wu and Chen's work is that TEM was used to analyze the nanoscale precipitate and crystal structure of the $\mathrm{AgSbTe}_{2}$ phase, which has promising thermoelectric properties.

Case study three: Determination of equilibrium compositions of disordered $\gamma$ (Fcc-A1) phase and ordered $\gamma^{\prime}\left(\mathrm{L1}_{2}\right)$ and $\theta$ $\left(\mathrm{DO}_{22}\right)$ phases at $800{ }^{\circ} \mathrm{C}$ in the Ni-Al-V system (Zapolsky et al., CALPHAD 25, 2001, p 125).

3DAP can provide three-dimensional maps of chemical heterogeneities at the Ångstrom-scale in materials. The Ni-Al-V ternary system is complicated since the disordered $\gamma$ phase and ordered $\gamma^{\prime}$ and $\theta$ phases coexist at elevated temperature and in Ni-rich compositions. It is problematic to use EPMA or SEM/EDX to determine the composition of these phases due to their nanosized grains. 3DAP was used to measure the equilibrium compositions of $\gamma$ disordered phase and ordered $\gamma^{\prime}$ and $\theta$ phases at $800{ }^{\circ} \mathrm{C}$ in the Ni-Al-V system. The measurements agree well with numerical calculations based on a microscopic mean field approach. Both the experimental measurements and calculations show that the equilibrium compositions of the coexisting phases are different from those indicated in the published phase diagrams (Hayes et al., in Ternary alloys, VCH, Weiheim, Germany, 1993, p 8).

Outlook: Based on the above three case studies, it should be highlighted that advanced experimental techniques, such as TEM and 3DAP, are providing us with more perspectives when exploring today's fascinating new materials, just as the invention of optical microscope opened for humans the gate to the micro-world in the 16th century. In the case of nanosystems, the size becomes an external parameter, like temperature and composition. Consequently, the Gibbs energy in the nanosystem is expressed as a function of temperature, composition and size. The use of TEM and 3DAP in conjunction with classical experimental techniques, such as DSC, could lead to the establishment of the nanophase diagram. When nanothermodynamic models are developed for nanophases, a CALPHAD-type thermodynamic optimization could be performed in order to obtain nanothermodynamic descriptions for nanosystems. It could take decades to accomplish nanothermodynamic databases for nanosystems as we have for bulk materials due to immense experimental difficulties to measure nanophase diagrams and severe challenge to develop physically sound nanothermodynamic models. In spite of this, I am still convinced that applying these cutting-edge technologies such as TEM and 3DAP in phase equilibrium determination new discoveries can be achieved.

\author{
Yong Du \\ Associate Editor \\ Journal of Phase Equilibria and Diffusion \\ Changsha, China \\ E-mail: yongducalphad@gmail.com
}

